\title{
Factores Pronósticos de Esquizofrenia en Primer Episodio Psicótico
}

\section{Prognostic factors for schizophrenia during first psychotic episode}

Juan F. Cano, Marco Fierro-Urresta, Claudia R. Vanegas, Marcela Alzate, Ana Olarte, Ricardo Cendales y Rodrigo N. Córdoba

Centro de investigaciones de investigaciones del sistema nervioso (CISNE)

rodrinel@yahoo.com,cisne@scientist.com

Recibido 29 Septiembre 2006/Enviado para Modificación 6 Mayo 2007/Aceptado 5 Julio

\section{RESUMEN}

Objetivo Revisar la literatura disponible sobre el pronóstico del primer episodio psicótico para el desarrollo de esquizofrenia.

Método Una revisión sistemática de los estudios que han evaluado los determinantes pronósticos para el primer episodio psicótico y su relación con esquizofrenia.

Resultados Se revisaron 161 artículos que cumplían con los criterios de búsqueda y que se ajustaban con el propósito del estudio.

Conclusiones El tiempo de psicosis no tratada (DUP), el nivel del funcionamiento premórbido, la presencia o predominio de síntomas negativos, el consumo comórbido de sustancias psicoactivas y el estado psicosocial fueron las características mas influyentes para el desarrollo de la esquizofrenia, en los pacientes que presentaban un primer episodio psicótico.

Palabras Clave: Esquizofrenia, trastornos psicóticos, pronóstico, diagnóstico precoz, (fuente: DeCS, BIREME).

\section{ABSTRACT}

Objective Reviewing the available literature regarding prognosis for first psychotic episode for developing schizophrenia.

Method A systematic review of studies which have evaluated prognostic determinants for the first psychotic episode and its relationship to schizophrenia was made.

Results 161 articles were reviewed which fulfilled the search criteria and which were adjusted to the purpose of the study.

Conclusions Duration of untreated psychosis (DUP), pre-morbid functioning level, the presence or predominance of negative symptoms, co-morbid consumption of psychoactive substances and psychosocial state were the most influential characteristics for developing schizophrenia in patients presenting a first psychotic episode. 
Key Words: Schizophrenia, psychotic disorder, prognosis, early diagnosis (source: $\mathrm{MeSH}, \mathrm{NLM})$.

$\mathrm{E}$ n el panorama epidemiológico, el Banco Mundial calcula que los trastornos neurológicos y psiquiátricos contribuyen con el $12 \%$ al costo total de las enfermedades médicas, y según la OMS con el 20\% (1). Estos padecimientos, medidos por años de vida ajustados en función de la discapacidad (AVAD), representan el $13 \%$ de la carga total de enfermedades (2). Entre los trastornos psicóticos la esquizofrenia tiene el peor pronóstico pues ocasiona deterioro en casi todas la funciones psicológicas y dificulta la adaptación social, académica y laboral. Su inicio y los cambios que conlleva pueden observarse como un deterioro lento y gradual $(3,4)$; en muchas ocasiones comienza como un primer episodio psicótico (PEP).

Se pretendía conocer respecto a la literatura mundial cuales son los factores determinantes del primer episodio psicótico, por lo que se llevo a cabo una revisión sistemática realizando una búsqueda en las bases de datos de PubMed, Embase y Ovid. Se incluyeron como palabras de búsqueda en todas las combinaciones posibles los términos MeSH (Medical Subject Heading): Schizophrenia, Psychotic Disorders, Diagnosis, Risk, Risk Assessment, Risk Factors y treatment. Se seleccionaron los artículos originales publicados desde enero de 1990 hasta julio de 2006 en los cuales se incluía el término: First episode. Se excluyeron aquellos estudios relacionados con trastorno afectivo bipolar, trastornos por consumo de sustancias y trastornos secundarios a condición médica general, hallándose un total de 161 artículos. Estos se evaluaron teniendo en cuenta metodología relevancia y actualidad, seleccionándose los que se encontraron pertinentes según la metodología propuesta. Se realizó un análisis descriptivo, anotando los factores reportados como determinantes del pronóstico en primer episodio psicótico, encontrándose especial relevancia en: Tiempo de psicosis no tratada, adaptación premórbida, predominio de síntomas negativos, consumo comórbido de sustancias psicoactivas y condiciones psicosociales asociadas. Se realiza al final una recopilación de las recomendaciones para el tratamiento del primer episodio psicótico.

\section{PRIMER EPISODIO PSICÓTICO}

La aparición abrupta, por primera vez en la vida, de un episodio de síntomas psicóticos se conoce como primer episodio psicótico (PEP). Es un cuadro de tipo sindromático que se aclara, en cuanto a un diagnóstico específico, sólo 
después de seguir la evolución clínica. Un buen porcentaje de los casos reciben, con el tiempo el diagnóstico de esquizofrenia. En cambio para otro grupo de pacientes, el PEP es el único episodio psicótico en su vida y no se acompaña de repercusiones en el funcionamiento psicosocial ni en la calidad de vida (5-7).

Según los criterios del DSM-IV, si los síntomas duran menos de un mes el diagnóstico es trastorno psicótico breve, entre un mes y seis meses: trastorno esquizofreniforme, más de seis meses: esquizofrenia. La duración no es el único criterio para determinar el pronóstico, ya que la remisión de los síntomas en menos de un mes, no siempre significa ausencia de recaídas (8).

En la actualidad, el estudio de las personas con trastornos psicóticos, se ha ampliado desde el ámbito meramente clínico hasta abarcar dimensiones del funcionamiento psicosocial como el empleo, la adaptación a las exigencias de la vida en comunidad y la calidad de vida (9-12). En los últimos años se han producido importantes avances terapéuticos; sin embargo, una proporción importante de pacientes termina con un resultado pobre en el mediano y largo plazo (5,9,13-16). Al parecer la mejoría a corto plazo no siempre se acompaña de la detención del proceso patológico subyacente.

\section{FACTORES QUE MODIFICAN EL CURSO Y PRONÓSTICO DEL PEP}

Se dispone de pocas evidencias sobre los factores que modifican del curso del $\mathrm{PEP}$, especialmente en lo relacionado con el efecto específico que sobre el desenlace en esquizofrenia tiene cada uno de ellos. El campo de investigación en intervenciones tempranas en psicosis es joven, especialmente cuando se busca factores que mejoren el resultado luego de que el primer episodio psicótico se ha presentado (17). Se ha planteado que el tiempo de psicosis no tratada (DUP), la adaptación premórbida, el predominio de síntomas negativos, el consumo comórbido de sustancias psicoactivas y las condiciones psicosociales, especialmente la pobreza se destacan como los más importantes en la determinación del pronóstico.

Tiempo de psicosis no tratada (DUP)

A partir de los estudios de Loebel, Lieberman y colaboradores (18) en Estados Unidos, de McGlashan $(19,20)$ en Noruega y el trabajo de McGorry (21) en Australia se modificó la manera de estudiar la esquizofrenia: mayor énfasis en la evaluación de las primeras fases de la enfermedad y en el diagnóstico. El tiempo de psicosis no tratada (DUP) hace referencia al periodo 
de tiempo transcurrido entre la aparición de los primeros síntomas psicóticos y el momento en el que se inicia el tratamiento. La duración de este periodo se ha relacionado con diferentes indicadores de remisión tales como recuperación global $(21,22)$, disfunción cognitiva $(23,25)$, severidad de los síntomas (26), respuesta al tratamiento $(27,28)$ y riesgo de recaída $(29,30)$. Perkins y colaboradores publicaron un meta análisis de la relación entre el DUP y el primer episodio psicótico (31). Revisaron 43 publicaciones de 28 centros y entre sus conclusiones afirman que un periodo prolongado de psicosis previo al comienzo de la terapia farmacológica se asocia con más bajos niveles de recuperación sintomática y funcional. Esta asociación es independiente del efecto de otras variables, y aunque su magnitud es ligera a moderada, se trata de un factor pronóstico potencialmente modificable. De allí la importancia de identificar tempranamente los síntomas y establecer un esquema terapéutico adecuado $(31,32)$.

Adaptación premórbida y pródromos

Los problemas en el funcionamiento o adaptación premórbida se consideran predictores del curso de la psicosis y se asocian con una instauración más temprana del deterioro $(8,19,28,33,34)$. Larsen relaciona el pobre funcionamiento premórbido con una mayor presencia de síntomas negativos como el aplanamiento afectivo, la apatía y la pobreza del pensamiento (34) Perkins encuentra que estos pacientes tienen pobre respuesta al tratamiento (28). Según Menezes y colaboradores, la adaptación premórbida del adolescente y la Escala Global del Funcionamiento (GAF) antes y después del PEP en adolescentes y adultos son los mejores predictores del curso de la psicosis, en consecuencia asignan al GAF un valor predictivo crucial en los dos tipos de poblaciones (33).

Se conoce que en los tres o cuatro años previos a la aparición del PEP se presentan una serie de síntomas psicológicos poco específicos y/o hay un declive en ciertas áreas del funcionamiento del individuo. A este conjunto de manifestaciones se le denomina pródromos. Los síntomas prodrómicos más conocidos son el afecto depresivo, el aislamiento social, la disminución de la concentración y motivación, las alteraciones del sueño y la suspicacia (35).

Síntomas semiológicos predominantes (psicopatología)

Andreasen propuso agrupar los síntomas de la esquizofrenia en positivos y negativos (36). Entre los primeros figuran los delirios, las alucinaciones, la desorganización del pensamiento y de la conducta. Los negativos, en cambio, 
son relativamente silenciosos y pueden pasar desapercibidos. Los principales son respuesta emocional disminuida, retraimiento social, apatía, desinterés en el entorno, pensamiento lento y empobrecido, así como falta de habilidades para el desempeño social. Los síntomas negativos parecen estar relacionados con un curso menos favorable de la enfermedad independientemente de la severidad de los síntomas positivos (33). Una vez establecido el episodio, la presencia temprana de síntomas negativos que no responden al tratamiento se relaciona con el diagnóstico de esquizofrenia y con un pobre resultado en términos de funcionalidad y calidad de vida (13).

En los pacientes con PEP se ha encontrado una menor introspección, lo que implica dificultad para atribuir los síntomas a una enfermedad e interpretar la experiencia psicótica como anormal. Como resultado hay menor aceptación y cumplimiento del tratamiento (37). Al igual que en los pacientes con esquizofrenia establecida, mientras mayor y mejor sea el conocimiento, y el reconocimiento de los síntomas, mejores los resultados (38).

Consumo de sustancias psicoactivas

El consumo de sustancias psicoactivas, especialmente el abuso, está presente en gran proporción de pacientes y es la principal comorbilidad en PEP (39). Es mayor al encontrado en la población general, en especial en lo referente al abuso de marihuana y alcohol $(40,41)$ y se ha relacionado con un peor pronóstico de la enfermedad y una mayor severidad de los síntomas. Cuando se brinda tratamiento el consumo disminuye notoriamente y no ejerce su efecto nocivo en el pronóstico a largo plazo (40).

\section{Condiciones psicosociales}

La prevalencia de la esquizofrenia es mayor en los estratos socioeconómicos bajos, pero no se ha encontrado una explicación clara al respecto. La situación se agrava debido a la dificultad en el acceso a los servicios de salud, al pobre reconocimiento de los síntomas prodrómicos por parte del personal médico, incluyendo a los psiquiatras, y al estigma que aún en países desarrollados genera la enfermedad mental (42).

\section{TRATAMIENTO}

Antes de iniciar el tratamiento específico con antipsicóticos se considera conveniente esperar entre 24 a 48 horas, con el fin de observar la evolución de 
los síntomas y descartar que el síndrome sea ocasionado por consumo de sustancias o por enfermedades médicas distintas a las psiquiátricas (43). Con base en estudios controlados se ha recomendado el uso de antipsicóticos tradicionales (también llamados típicos o de primera generación) para el manejo del PEP en jóvenes (44). Hay reportes de buenos resultados con dosis bajas de Haloperidol (45-47) especialmente si se combinan con un adecuado apoyo psicosocial (46). Sin embargo, la aparición de efectos secundarios como los síntomas extrapiramidales ha llevado a plantearse la posibilidad de utilizar nuevos antipsicóticos como primera línea de tratamiento (44).

En cuanto al papel de los antipsicóticos atípicos (o de segunda generación) los datos hasta ahora disponibles arrojan resultados esperanzadores ya que producen menos efectos extrapiramidales y se asocian con menor riesgo de disquinesia tardía, lo que ayuda a una mejor adherencia al tratamiento (48).

Los antipsicóticos atípicos hasta el momento estudiados en el manejo del PEP son Clozapina, Olanzapina y Risperidona. La Clozapina produce pocos síntomas extrapiramidales, si bien efectos secundarios como sedación, aumento de peso, hipotensión ortostática y taquicardia han sido observado en los adolescentes(49). Woerner y colaboradores no encontraron un beneficio adicional con este medicamento especialmente por la alta proporción de pacientes que descontinuaron el tratamiento con este medicamento (50). En cuanto a la Olanzapina aunque produce buenos resultados en adultos, aún faltan datos sobre eficacia y efectos adversos en adolescentes (49). En la revisión de Duggan se concluye que ofrece un buen efecto antipsicótico, menos síntomas extrapiramidales que los medicamentos típicos, pero con mayor ganancia de peso (51). Los estudios con Risperidona indican que posee eficacia similar a los antipsicóticos tradicionales en adultos y buenos resultados en adolescentes que no toleran o no responden al tratamiento con éstos.

Comparados con los de primera generación, los de segunda poseen un efecto terapéutico igual o mayor, producen menos síntomas extrapiramidales $(44,52)$ pero se asocian con alteraciones endocrinas y metabólicas como aumento de peso, de glicemia y de colesterol, así como mayor riesgo de desarrollar diabetes tipo II, por lo que algunos autores plantean que no se deben descartar de plano los medicamentos clásicos (53).

\section{CONCLUSIONES}

No es fácil predecir el curso que tomará el primer episodio psicótico: ¿Se trata de un episodio único en la vida o el comienzo de una esquizofrenia? Entre los factores 
estudiados se ha encontrado que el tiempo transcurrido de síntomas sin recibir tratamiento, la precariedad en el funcionamiento previo, el consumo de sustancias y la aparición temprana de síntomas negativos indican un peor pronóstico y se constituyen en factores de riesgo para la aparición de la esquizofrenia

\section{REFERENCIAS}

1. Murray CJ, Lopez AD. The global burden of disease and injury series, volume 1: a comprehensive assessment of mortality and disability from diseases, injuries, and risk factors in 1990 and projected to 2020. Cambridge, MA: Harvard University Press; 1996.

2. Beaglehole R, Irwin A, Prentice T, Informe sobre la salud en el mundo 2003: Forjemos el futuro. OMS; 2003. Report No.: 9.

3. Meltzer HY, Hossein-Fatemi S. Schizophrenia. In: Ebert MH, Loosen PT, Nurcombe B, editors. Current diagnosis \& treatment in psychiatry; 2006.

4. Lauriello J, Bustillo JR, Keith SJ. Schizophrenia: Scope of the Problem. In: Sadock BJ, Sadock VA, editors. Kaplan \& Sadock's Comprehensive Textbook of Psychiatry. 8 ed. Philadelphia: Lippincott Williams \& Wilikins; 2003.

5. Robinson DG Woerner MG McMeniman M, Mendelowitz A, Bilder RM. Symptomatic and functional recovery from a first episode of schizophrenia or schizoaffective disorder. Am J Psychiatry 2004 Mar;161(3):473-9.

6. Singh SP, Croudace T, Amin S, Kwiecinski R, Medley I, Jones PB, et al. Three-year outcome of first-episode psychoses in an established community psychiatric service. Br J Psychiatry 2000 Mar;176:210-6.

7. Zhang-Wong J, Beiser M, Bean G, Iacono WG. Five-year course of schizophreniform disorder. Psychiatry Res 1995 Nov 29;59(1-2):109-17.

8. Pillmann F, Haring A, Balzuweit S, MarnerosA. A comparison of DSM-IV brief psychotic disorder with "positive" schizophrenia and healthy controls. Compr Psychiatry 2002 Sep;43(5):385-92.

9. Rosenheck R, Leslie D, Keefe R, McEvoy J, Swartz M, Perkins D, et al. Barriers to employment for people with schizophrenia. Am J Psychiatry 2006 Mar;163(3):4117.

10. Freeman H., Katschnig H., H.Sartorius. Calidad de Vida en los Trastornos mentales. Barcelona: Masson; 2000.

11. Law CW, Chen EY, Cheung EF, Chan RC, Wong JG, Lam CL, et al. Impact of untreated psychosis on quality of life in patients with first-episode schizophrenia. Quality of Life Research 2005Oct;14(8):1803-11.

12. Russo J, Roy-Byrne P, Jaffe C, Ries R, Dagadakis C, Avery D. Psychiatric status, quality of life, and level of care as predictors of outcomes of acute inpatient treatment. Psychiatric Services 1997;48(11):1427-34. 
13. Malla A, Payne J. First-episode psychosis: psychopathology, quality of life, and functional outcome. Schizophr Bull 2005 Jul;31(3):650-71.

14. Birchwood M, Todd P, Jackson C. Early intervention in psychosis. The critical period hypothesis. Br J Psychiatry Suppl 1998;172(33):53-9.

15. Larsen TK, McGlashan TH, Moe LC. First-episode schizophrenia: I. Early course parameters. Schizophr Bull 1996;22(2):241-56.

16. Penn DL, Waldheter EJ, Perkins DO, Mueser KT, Lieberman JA. Psychosocial treatment for first-episode psychosis: a research update. Am J Psychiatry 2005 Dec;162(12):2220-32.

17. Malla AK, Norman RM, Joober R. First-episode psychosis, early intervention, and outcome: what have we learned? Can J Psychiatry 2005 Dec;50(14):881-91.

18. Loebel AD, Lieberman JA, Alvir JM, MayerhoffDI, Geisler SH, Szymanski SR. Duration of psychosis and outcome in first-episode schizophrenia. Am J Psychiatry 1992 Sep;149(9):1183-8.

19. Larsen TK, Johannessen JO, Opjordsmoen S. First-episode schizophrenia with long duration of untreated psychosis. Pathways to care. Br J Psychiatry Suppl 1998;172(33):45-52.

20. McGlashan TH. Duration of untreated psychosis in first-episode schizophrenia: marker or determinant of course? Biol Psychiatry 1999 Oct 1;46(7):899-907.

21. McGorry PD. The influence of illness duration on syndrome clarity and stability in functional psychosis: does the diagnosis emerge and stabilise with time? Aust N Z J Psychiatry 1994 Dec;28(4):607-19.

22. Black K, Peters L, Rui Q, Milliken H, Whitehorn D, Kopala LC. Duration of untreated psychosis predicts treatment outcome in an early psychosis program. Schizophr Res 2001 Mar 1;47(2-3):215-22.

23. Hoff AL, Riordan H, O'Donnell DW, Morris L, DeLisi LE. Neuropsychological functioning of first-episode schizophreniform patients. Am J Psychiatry 1992 Jul;149(7):898-903.

24. Malla AK, Norman RM, Manchanda R, Townsend L. Symptoms, cognition, treatment adherence and functional outcome in first-episode psychosis. Psychol Med 2002Aug;32(6):1109-19.

25. Joyce E, Hutton S, Mutsatsa S, Gibbins H, Webb E, Paul S, et al. Executive dysfunction in first-episode schizophrenia and relationship to duration of untreated psychosis: the West London Study. Br J Psychiatry Suppl 2002 Sep;43:s38-s44.

26. Kalla O, Aaltonen J, Wahlstrom J, Lehtinen V, Garcia C, I, Gonzalez de CM. Duration of untreated psychosis and its correlates in first-episode psychosis in Finland and Spain. Acta Psychiatr Scand 2002 Oct;106(4):265-75.

27. Barnes TR, Hutton SB, Chapman MJ, Mutsatsa S, Puri BK, Joyce EM. West London first-episode study of schizophrenia. Clinical correlates of duration of untreated psychosis. Br J Psychiatry 2000 Sep;177:207-11. 
28. Perkins D, Lieberman J, Gu H, Tohen M, McEvoy J, Green A, et al. Predictors of antipsychotic treatment response in patients with first-episode schizophrenia, schizoaffective and schizophreniform disorders. Br J Psychiatry 2004 Jul;185:1824.

29. Wiersma D, Nienhuis FJ, Slooff CJ, Giel R. Natural course of schizophrenic disorders: a 15-year followup of a Dutch incidence cohort. Schizophr Bull 1998;24(1):75-85.

30. Verdoux H, Liraud F, Bergey C, Assens F, Abalan F, van OJ. Is the association between duration of untreated psychosis and outcome confounded? A two year followup study of first-admitted patients. Schizophr Res 2001 Apr 30;49(3):231-41.

31. Perkins DO, Gu H, Boteva K, Lieberman JA. Relationship between duration of untreated psychosis and outcome in first-episode schizophrenia: a critical review and meta-analysis. Am J Psychiatry 2005 Oct;162(10):1785-804.

32. Kohn D, Pukrop R, Niedersteberg A, Schultze-Lutter F, Ruhrmann S, BechdolfA, et al. [Pathways to care: help-seeking behavior in first-episode psychosis]. Fortschr Neurol Psychiatr 2004 Nov;72(11):635-42.

33. Menezes NM, Milovan E. First-episode psychosis: a comparative review of diagnostic evolution and predictive variables in adolescents versus adults. Can J Psychiatry 2000 Oct;45(8):710-6.

34. Larsen TK, Moe LC, Vibe-Hansen L, Johannessen JO. Premorbid functioning versus duration of untreated psychosis in 1 year outcome in first-episode psychosis. Schizophr Res 2000 Sep 29;45(1-2):1-9.

35. Escobar R. Primer episodio psicótico. Avances en psiquiatría biológica 2000;1:46-55.

36. Andreasen NC, Flaum M. Schizophrenia: the characteristic symptoms. Schizophr Bull 1991;17(1):27-49.

37. Novak-Grubic V, Tavcar R. Predictors of noncompliance in males with first-episode schizophrenia, schizophreniform and schizoaffective disorder. Eur Psychiatry 2002 May;17(3):148-54.

38. Thompson KN, McGorry PD, Harrigan SM. Reduced awareness of illness in firstepisode psychosis. Compr Psychiatry 2001 Nov;42(6):498-503.

39. Strakowski SM, Tohen M, Stoll AL, Faedda GL, Mayer PV, Kolbrener ML, et al. Comorbidity in psychosis at first hospitalization. Am J Psychiatry 1993 May;150(5):752-7.

40. Lambert M, Conus P, Lubman DI, Wade D, Yuen H, Moritz S, et al. The impact of substance use disorders on clinical outcome in 643 patients with first-episode psychosis. Acta Psychiatr Scand 2005 Aug;112(2):141-8.

41. Wade D, Harrigan S, Edwards J, Burgess PM, Whelan G, McGorry PD. Course of substance misuse and daily tobacco use in first-episode psychosis. Schizophr Res2006 Jan 31;81(2-3):145-50. 
42. Royal Australian and New Zealand College of Psychiatrists clinical practice guidelines for the treatment of schizophrenia and related disorders. Aust N Z J Psychiatry 2005Jan;39(1-2):1-30.

43. Lambert M, Conus P, Lambert T, McGorry PD. Pharmacotherapy of first-episode psychosis. Expert Opin Pharmacother 2003 May;4(5):717-50.

44. Walter G, Wiltshire C, Anderson J, Storm V. The pharmacologic treatment of the early phase of first-episode psychosis in youths. Can J Psychiatry 2001 Nov;46(9):8039.

45. Apiquian R, Fresan A, Herrera K, Ulloa RE, Loyzaga C, de IF-S, et al. Minimum effective doses of haloperidol for the treatment of first psychotic episode: a comparative study with risperidone and olanzapine. Int J Neuropsychopharmacol 2003Dec;6(4):403-8.

46. Cullberg J, Levander S, Holmqvist R, Mattsson M, Wieselgren IM. One-year outcome in first episode psychosis patients in the Swedish Parachute project. Acta Psychiatr Scand 2002 Oct;106(4):276-85.

47. Zhang-Wong J, Zipursky RB, Beiser M, Bean G. Optimal haloperidol dosage in firstepisode psychosis. Can J Psychiatry 1999 Mar;44(2):164-7.

48. Van Os J, Andreasen NC, Flaum M. From first episode to long-term care: The need for sustained clinical commitment. International Journal of Psychiatry in Clinical Practice 2000;4(S):19-24.

49. Lewis R. Typical and atypical antipsychotics in adolescent schizophrenia: efficacy, tolerability, and differential sensitivity to extrapyramidal symptoms. Can J Psychiatry 1998 Aug;43(6):596-604.

50. Woerner MG Robinson DG, Alvir JM, Sheitman BB, Lieberman JA, Kane JM. Clozapine as a first treatment for schizophrenia. Am J Psychiatry 2003 Aug;160(8):1514-6.

51. Duggan L, Fenton M, Rathbone J, Dardennes R, El-Dosoky A, Indran S. Olanzapine for schizophrenia. Cochrane Database Syst Rev 2005;(2):CD001359.

52. Bradford DW, Perkins DO, Lieberman JA. Pharmacological management of firstepisode schizophrenia and related nonaffective psychoses. Drugs 2003;63(21):2265-83.

53. Gardner DM, Baldessarini RJ, Waraich P. Modern antipsychotic drugs: a critical overview. CMAJ 2005 Jun 21;172(13):1703-11. 